\title{
Menschenwürde: Ein für die Medizinethik irrelevanter Begriff?
}

\author{
Schaber, Peter
}

\begin{abstract}
Es wurde für die These argumentiert, dass der Begriff der Würde in der Medizinethik nutzlos sei und in den Fällen, in denen er verständlich verwendet wird, nichts anderes meint als den Respekt vor der Autonomie von Personen. In diesem Aufsatz soll gezeigt werden, dass diese These falsch ist. Es wird ein Begriff von Würde vorgestellt, der sich nicht auf den Begriff des Respekts vor der Autonomie von Personen reduzieren lässt. Anhand der Diskussion um ein Sterben in Würde soll auch deutlich werden, dass auf den Begriff der Würde auch in der Medizinethik nicht verzichtet werden kann. Definition of the problem: It has been argued that the concept of dignity is of no use in medical ethics and that it means - in cases where we know what is meant by it - nothing but respect for the autonomy of persons. Arguments: It is the aim of this paper to show that this claim is false and should be rejected. A certain idea of dignity is developed that can be distinguished from respect for the autonomy of persons. This concept is applied to the discussion about the idea of dying with dignity. Conclusion: The concept of dignity cannot be replaced by the concept of autonomy.
\end{abstract}

DOI: https://doi.org/10.1007/s00481-012-0218-4

Posted at the Zurich Open Repository and Archive, University of Zurich ZORA URL: https://doi.org/10.5167/uzh-69336

Journal Article

Published Version

Originally published at:

Schaber, Peter (2012). Menschenwürde: Ein für die Medizinethik irrelevanter Begriff? Ethik in der Medizin, 24(4):297-306.

DOI: https://doi.org/10.1007/s00481-012-0218-4 


\title{
Menschenwürde: ein für die Medizinethik irrelevanter Begriff?
}

\author{
Peter Schaber
}

Online publiziert: 14. Oktober 2012

(C) Springer-Verlag 2012

Zusammenfassung Es wurde für die These argumentiert, dass der Begriff der Würde in der Medizinethik nutzlos sei und in den Fällen, in denen er verständlich verwendet wird, nichts anderes meint als den Respekt vor der Autonomie von Personen. In diesem Aufsatz soll gezeigt werden, dass diese These falsch ist. Es wird ein Begriff von Würde vorgestellt, der sich nicht auf den Begriff des Respekts vor der Autonomie von Personen reduzieren lässt. Anhand der Diskussion um ein Sterben in Würde soll auch deutlich werden, dass auf den Begriff der Würde auch in der Medizinethik nicht verzichtet werden kann.

Schlüsselwörter Würde $\cdot$ Autonomie $\cdot$ Achtung vor der Autonomie $\cdot$ Sterben in Würde

\section{Is dignity a useless concept in medical ethics?}

Abstract Definition of the problem It has been argued that the concept of dignity is of no use in medical ethics and that it means - in cases where we know what is meant by it - nothing but respect for the autonomy of persons. Arguments It is the aim of this paper to show that this claim is false and should be rejected. A certain idea of dignity is developed that can be distinguished from respect for the autonomy of persons. This concept is applied to the discussion about the idea of dying with dignity. Conclusion The concept of dignity cannot be replaced by the concept of autonomy.

Keywords Dignity $\cdot$ Autonomy $\cdot$ Respect for autonomy $\cdot$ Dying with dignity 
Wer von der Würde des Menschen redet, muss mit dem Vorwurf rechnen, einen Begriff zu verwenden, der keine Bedeutung hat und auf den man entsprechend besser ersatzlos verzichten sollte. Für einige ist der Begriff der Würde nicht nur leer, sondern auch in Gefahr, für beliebige ideologische Zwecke benutzt zu werden. Wem eine bestimmte Praxis missfällt, der aber kein Argument dagegen vorzubringen weiß, bezeichnet diese - so wird gesagt - einfach als eine Verletzung der Menschenwürde. ${ }^{1}$ Gerade weil der Begriff leer sei und beliebig besetzt werden könne, eigne er sich als Mittel für unterschiedlichste ideologische Zwecke.

Es wird auch die Ansicht vertreten, der Begriff der Menschenwürde sei nicht leer, sondern einfach ersatzlos durch andere Begriffe zu ersetzen. Anstatt von „Menschenwürde“ sollten wir, wie Norbert Hoerster meint, von „Menschenrechten“ ' reden. ${ }^{2}$ Eine ähnliche Redundanzthese wird auch von der englischen Bioethikerin Ruth Macklin vertreten [6]. Dort, wo in der Medizinethik von Menschenwürde gesprochen werde, sei, so Macklin, oft nichts anderes als die Achtung vor der Autonomie von Personen gemeint:

References to dignity emerged in the 1970s in discussions about the process of dying, in particular, the desire to avoid burdensome life prolonging treatment [...] In this context dignity seems to be nothing other than respect for autonomy ([6], S. 1419).

Dabei gibt es nach Macklin auch Verwendungen des Begriffs, bei denen etwas anderes gemeint sei als Achtung vor der Autonomie von Personen. In den Fällen wüsste man allerdings nicht, was genau gemeint sei. Deshalb rät Macklin dazu, sich auf die eine klare Verwendungsweise des Würdebegriffs zu beschränken und statt von „Würde“ von der „Achtung vor der Autonomie von Personen“" zu reden.

Macklins Redundanzthese ist der Gegenstand der nachfolgenden Ausführungen. Sollten wir - so lautet die Frage - anstatt von Würde des Menschen von der Achtung vor der Autonomie von Personen reden? Oder hat der Würdebegriff eine gegenüber dem Autonomiebegriff eigenständige normative Bedeutung? Und handelt es sich, wenn es sich um einen eigenständigen normativen Begriff handelt, deshalb um einen Begriff, auf den auch in der Medizinethik nicht verzichtet werden kann? Es sollen nachfolgend einige Gründe genannt werden, weshalb der Begriff der Würde nicht durch den Begriff der Achtung vor der Autonomie von Personen ersetzt werden kann. Anhand der Diskussion um ein Sterben in Würde, soll auch deutlich werden, dass auf den Begriff der Würde auch in der Medizinethik nicht verzichtet werden kann. Der Würdebegriff besitzt einen eigenen normativen Gehalt und sollte deshalb nicht aufgegeben werden.

\section{Ohne Bedeutung?}

Bevor ich allerdings auf die eigenständige normative Bedeutung des Würdebegriffs eingehe, möchte ich mich mit dem Vorwurf, der Begriff sei leer, befassen. Im Unterschied zur Redundanzthese meint, wer den Begriff für leer hält, nicht, dass er durch einen anderen ersetzt werden kann, sondern dass er schlicht keine bestimmbare Bedeutung besitzt.

\footnotetext{
${ }^{1}$ So z. B. Hoerster [4], S. 24: Man findet, ,im Begriff der Menschenwürde die geradezu ideale Waffe [...] In Wirklichkeit hat man jedoch nichts begründet, sondern seiner Bewertung lediglich auf besonders suggestive Weise Ausdruck gegeben."

${ }^{2}$ Hoerster [4], S. 25: „So... kann man sagen, dass das Prinzip der Menschenwürde in seiner rechtlichen Bedeutung in einem einzigen Begriff zusammenfasst, was die verschiedenen Menschenrechte gemeinsam beinhalten."
} 
Dieser Vorwurf wird i.d.R. mit dem Hinweis begründet, der Würdebegriff werde in sehr unterschiedlicher Weise verwendet. Ist dieser Umstand ein Beleg dafür, dass es sich um einen leeren Begriff handelt? Zunächst teilt der Begriff der Würde mit anderen Begriffen das Schicksal, unterschiedlich verwendet und verstanden zu werden. Unter Gerechtigkeit wird Unterschiedliches verstanden, genauso unter dem Begriff der Legitimität, der Chancengleichheit und dem der moralischen Rechte. Das wäre im Vergleich zur Verwendung des Begriffs der Menschenwürde bloß dann unproblematisch, wenn in Bezug auf die genannten Begriffe der Beliebigkeit Grenzen gesetzt werden könnten, die sich für den Begriff der Menschenwürde nicht formulieren ließen. Solche Grenzen lassen sich aber auch für den Begriff der Menschenwürde durchaus formulieren.

Bestimmte Handlungen würde niemand als eine Verletzung der Würde von Menschen bezeichnen. Wenn A sich weigert, B ein Buch auszuleihen, wird darin niemand eine Verletzung von A's Würde sehen. Und wir würden z. B. auch nicht sagen, dass Nicolas Sarkozy in seiner Würde verletzt worden ist, weil er nicht mehr zum Präsidenten Frankreichs gewählt worden ist. Zudem gibt es paradigmatische Fälle von Würdeverletzungen. In seiner Würde verletzt wird z. B. jemand, der in aller Öffentlichkeit gedemütigt wird. Auch bei Folter oder massiven Formen der Diskriminierung reden wir von der Verletzung der Würde. Hoerster gesteht dies zu, wendet aber ein: „(D)adurch, dass wir Beispiele anführen, die unter einen Begriff fallen, haben wir diesen Begriff ja noch nicht definiert“" ([4], S. 12).

Hier liegt allerdings ein Missverständnis darüber vor, wie man zu Begriffsbestimmungen gelangt. Wir tun dies nämlich generell, indem wir die Weise, wie ein Begriff verwendet wird, analysieren. Das gilt für alle Begriffe, nicht bloß für den Begriff der Würde. Wenn wir z. B. wissen wollen, was wir mit dem Ausdruck ,freiwillig“ meinen, müssen wir prüfen, wie dieser Begriff verwendet wird, genauer: welche Handlungen als paradigmatische Fälle freiwilliger Handlungen gesehen werden. Das verhält sich mit dem Begriff der Menschenwürde nicht anders: Wenn wir wissen wollen, was mit Menschenwürde gemeint ist, müssen wir auf die Verwendung schauen, insbesondere meiner Ansicht nach darauf, was wir als Verletzung der Würde bezeichnen. Daraus lassen sich Vorschläge für ein allgemeines Verständnis entwickeln, das unseren zentralen Verwendungsweisen Rechnung trägt und uns gleichzeitig in die Lage versetzt, gewisse Verwendungsweisen als unangemessene zurückzuweisen. Es ist nicht ersichtlich, wieso einer solchen Analyse des Würdebegriffs weniger Erfolg beschieden sein soll als der Analyse anderer Begriffe. Die Tatsache, dass der Menschenwürdebegriff unterschiedlich verwendet wird, begründet deshalb nicht die Forderung, auf die Verwendung des Begriffs zu verzichten. Sie legt lediglich eine nähere Bestimmung des Begriffs nahe, für deren Scheitern kein a priori Grund zu sehen ist. Dabei ist klar, dass der Begriff einer präzisen Bestimmung bedarf. Nur so lässt sich vermeiden, dass der Begriff zu unterschiedlichen praktischen Zwecken verwendet wird. Die Analyse des Begriffs sollte seine große normative Bedeutung deutlich machen. Zugleich sollte die Begriffsbestimmung eine plausible Interpretation dessen darstellen, was wir unter dem Begriff der Würde in der Allgemeinen Menschenrechtserklärung (Präambel: „Alle Menschen sind frei und gleich an Würde und Rechten geboren") und in den verschiedenen Verfassungen (Art. 1 des deutschen Grundgesetzes: „Die Würde des Menschen ist unantastbar“") angesprochen finden.

\section{Würde als Achtung der Autonomie?}

Die Begriffsanalyse könnte auch ergeben, dass der Begriff der Würde sich von einem anderen Begriff nicht unterscheiden lässt. Das ist die Idee der Redundanzthesen, unter anderem auch derjenigen von Ruth Macklin, die besagt, Würde ließe sich auf Achtung vor der Auto- 
nomie von Personen reduzieren. Macklin begründet die These damit, dass in medizinethischen Kontexten mit dem Begriff der Würde oft nur dies gemeint sei. Im Blick auf eine Aussage zur Würde des Menschen, die sich im Bericht des Nuffield Council on Bioethics zum Thema „Genetics, Freedom and Human Dignity“ findet, meint Macklin:

Although this renders the concept of human dignity meaningful, it is nothing more than a capacity for rational thought and action, the central features conveyed in the principle of respect for autonomy ([6], S. 1420).

Wenn z.B. von einem Sterben in Würde die Rede sei, stehe ein selbstbestimmtes Sterben im Blick. Manchmal sei allerdings auch unklar, was mit Würde gemeint sei. Der Begriff der Würde könnte immer, wenn er verständlich verwendet wird, durch den Begriff des Respekts vor der Autonomie ersetzt werden, ohne dass sich an den Aussagen etwas ändern würde. Der Begriff der Würde sei deshalb irreführend: Er stifte Verwirrung, weil er suggeriere, es stehe etwas anderes als der Respekt vor der Autonomie von Personen im Blick. Was dieses andere sei, könne aber niemand wirklich sagen. Die These, die hier zur Diskussion steht, lautet also: In jedem Fall, wo von Würde die Rede ist, kann man auch ohne Bedeutungsverlust von Achtung vor der Autonomie von Personen sprechen. Würde meint nicht bloß in einigen, sondern in allen Fällen Achtung vor der Autonomie.

Nun könnte man das, was Macklin hier vorschlägt, einfach als eine Explikation des Begriffs der Würde verstehen. ${ }^{3}$ Eine Explikation macht einen Begriff nicht überflüssig. Mit der Redundanzthese kann nicht gemeint sein, dass der Begriff der Würde durch andere Begriffe wie den der Autonomie expliziert werden kann. Dann wären alle Begriffe, die definiert werden, redundant. Mit der Redundanzthese muss deshalb etwas anderes gemeint sein. Macklins Explikation des Würdebegriffs sagt uns nicht, was wir üblicherweise mit Würde meinen. Wäre dies der Fall, läge einfach eine Explikation des Würdebegriffs vor, welche diesen genauso wenig überflüssig macht, wie eine beliebige Explikation eines Begriffs dies tut. Als Redundanzthese kann Macklins Vorschlag nur dann verstanden werden, wenn wir davon ausgehen, dass wir Würde nicht einfach als Achtung vor der Autonomie von Personen verstehen. Macklins Überlegung scheint dabei die folgende zu sein: In den Fällen, in denen der Würdebegriff in einer verständlichen Weise verwendet wird, ist mit ihm der Respekt vor der Autonomie gemeint. Der Würdebegriff wird aber oft in anderer Weise gebraucht. Wir wissen nicht, was in den anderen Fällen gemeint ist. Genau deshalb stiftet der Begriff der Würde Verwirrung: In den Fällen, in denen er nicht im Sinne der Achtung vor der Autonomie von Personen verstanden wird, ist nicht klar, wovon die Rede ist. Um diese Verwirrung zu vermeiden, sollten wir auf ihn verzichten und in Zukunft bloß noch von der Achtung vor der Autonomie reden.

Sollten wir das allerdings wirklich tun? Um diese Frage zu beantworten, müssen wir vorgängig wissen, was es heißt, die Autonomie einer Person zu achten. Der Autonomiebegriff ist selber umstritten [9]. Es ist deshalb alles andere als klar, was mit Würde gemeint wäre, würden wir darunter die Achtung vor der Autonomie von Personen verstehen. Wir bräuchten also vorgängig eine Klärung des Ausdrucks „Achtung vor der Autonomie von Personen“. Und das wäre wiederum das Thema einer eigenen Abhandlung.

Was man zeigen kann, ist dies, wie sich bestimmte Auffassungen von Autonomie und Würde voneinander unterscheiden. Das ist es auch, was ich im Blick auf ein wichtiges Verständnis der Achtung vor der Autonomie deutlich machen möchte. An einem prominenten Vorschlag zum Verständnis des Respekts vor der Autonomie soll gezeigt werden, wie

${ }^{3}$ Für in diesem Zusammenhang klärende Diskussionen möchte ich Holger Baumann und Sebastian Muders danken. 
Würde und Respekt vor der Autonomie sich unterscheiden könnten. Der Vorschlag, den ich hier im Blick habe, stammt vom Rechtsphilosophen Joel Feinberg. Feinberg schlägt vor, den Respekt vor der Autonomie in folgender Weise zu verstehen:

(R)espect for a person's autonomy is respect for his unfettered voluntary choice as the sole rightful determinant of his actions except where the interests of others need protection from him ([2], S.68).

Wenn Macklin nun Recht hätte, würde eine Person in ihrer Würde zu achten, eben dies bedeuten: sie nicht daran zu hindern, das zu tun, was sie tun will, und sie in ihren Entscheidungen zu achten, sofern sie freiwillig handelt und dabei niemandem Schaden zufügt. Achtung vor der Autonomie einer Person ist so verstanden Achtung vor den freiwilligen Entscheidungen einer Person. Ist dies mit den klaren Verwendungsweisen von Würde gemeint?

Es gibt verschiedene Gründe, das zu bestreiten. Die Würde des Menschen wird als ein grundlegendes Gut aufgefasst, dessen Verletzung schwerwiegend ist. Die Formulierung des deutschen Grundgesetzes, die Würde sei unantastbar, legt nahe, nicht nur, dass die Würde nie verletzt werden darf, sondern auch dass deren Verletzung ein schwerwiegendes moralisches Vergehen darstellt. Mit der Würde wird nach Artikel 1 des deutschen Grundgesetzes gar das grundlegendste Gut der Rechtsgemeinschaft verletzt. Wenn mit Würde die Achtung vor der Autonomie gemeint wäre, müsste das auch auf jede Missachtung, also Verletzung der Autonomie zutreffen. Das ist aber wenig plausibel. Nehmen wir an, ich würde daran gehindert, ein Stück Kuchen zu kaufen. Das wäre eine Missachtung meiner Autonomie und entsprechend auch moralisch falsch. Niemand würde darin allerdings ein schwerwiegendes moralisches Vergehen, gar die Verletzung des grundlegendsten Rechtsguts sehen. Niemand würde das als eine Verletzung meiner Würde betrachten. Autonomieverletzungen sind also ganz offensichtlich nicht immer Verletzungen der Würde von Menschen.

Man könnte sagen, dass nicht jede Autonomieverletzung auch eine Würdeverletzung, aber jede Würdeverletzung eine Autonomieverletzung darstellt. Muss Macklins Redundanzthese so verstanden werden? Betrachten wir dazu eine paradigmatische Form der Würdeverletzung. In ihrer Würde verletzt wird eine Person, wenn sie von anderen gedemütigt wird ([7], S. 9 ff.). Was - so ist zu fragen - macht Demütigungen zu Würdeverletzungen? Es ist meiner Ansicht nach Folgendes: Wer gedemütigt wird, wird von anderen herabgesetzt und nicht als Gleicher anerkannt. Wer gedemütigt wird, dem wird klar gemacht, dass man über ihn verfügen kann, dass er zur Disposition der anderen steht. Die Würdeverletzung, so könnte man sagen, besteht also darin, dass dem Gedemütigten das Recht abgesprochen wird, anderen als Gleicher gegenüberzutreten, der über sein Leben selber bestimmen kann. Würde haben heißt, als Gleicher anerkannt zu sein, über dessen Leben nicht verfügt werden darf, der darüber vielmehr selbst verfügt und entsprechend als jemand anerkannt ist, der eine normative Autorität über das eigene Leben besitzt (vgl. [8], S. 59 ff.). Demütigungen verletzen auch die Autonomie von Personen. Sie tun aber mehr als dies: Sie verletzen das Recht von Personen in gleicher Weise wie alle anderen, unabhängig von Geschlecht, Rasse, ethnischer und kultureller Zugehörigkeit, über ihr eigenes Leben verfügen zu dürfen.

Was allerdings heißt es, normative Autorität über sich selbst zu haben? Damit ist keine Fähigkeit gemeint, sondern das Recht, bestimmen zu können, was mit der eigenen Person getan wird. Das ist ein Recht, das mit der Pflicht der anderen korrespondiert, nicht in meine essentiellen Lebensbereiche einzugreifen. Zur normativen Autorität über sich selbst gehört i. d. R. auch das Recht, andere von den genannten Pflichten fallweise zu entbinden. Betrachten wir das an einem Bereich, über den ich normative Autorität besitze: meinen eigenen Körper. Ich habe das Recht, andere von der Benutzung meines Körpers auszuschließen. Die anderen haben entsprechend die Pflicht, von einer solchen Nutzung abzusehen. Dies 
jedenfalls solange, als ich sie nicht von ihrer Pflicht befreie. So habe ich z.B. das Recht, anderen zu erlauben, mich zu berühren. Diese Erlaubnis haben andere aber nur solange, als ich sie aus ihrer Pflicht entlasse. Es gehört zu meinem Recht über den eigenen Körper, ihnen diese Erlaubnis wieder zu entziehen. Die normative Autorität über den eigenen Körper ist in diesem Sinne ein Bündel von Rechten: das Recht, von anderen zu fordern, den meinen Körper nicht zu benutzen, und das Recht, sie von dieser Pflicht fallweise zu entbinden. Diese Rechte über meinen eigenen Körper sind ein Beispiel für die normative Autorität, die ich über mich selbst habe. Eine normative Autorität habe ich nicht bloß über meinen Körper, sondern über sämtliche Bereiche, die für mein Leben von essentieller Bedeutung sind. So habe ich das Recht zu bestimmen, mit wem ich zusammenlebe, welchen Beruf ich ausübe, welcher Partei ich angehöre etc.

$\mathrm{Zu}$ dieser normativen Autorität über sich selbst gehört es auch, sie ausüben zu können. Das setzt voraus, dass man im Blick auf essentielle Bereiche des eigenen Lebens zwischen akzeptablen Optionen wählen kann. Wenn ich ein Recht habe, zu bestimmen, welchen Beruf ich ausübe, muss ich die Wahl zwischen Berufen habe, die zu wählen ich Gründe habe. Es gehört zur Möglichkeit, die normative Autorität über sich ausüben zu können, zwischen akzeptablen Optionen wählen zu können.

Was unterscheidet - so lässt sich fragen - die normative Autorität über sich von der Autonomie einer Person? Die normative Autorität einer Person zu achten, heißt, ihr ein Recht zuzugestehen, über essentielle Bereiche ihres Lebens bestimmen zu können. Die normative Autorität einer Person wird entsprechend missachtet, wenn sie erniedrigt und gedemütigt wird, ${ }^{4}$ wenn man ihr klar machen will, dass sie nicht zählt, dass man mit ihr machen kann, was man will, dass sie ein Objekt ist, über das man beliebig verfügen kann. Wenn in dieser Weise die normative Autorität von Personen missachtet wird, wird auch die Autonomie von Personen nicht geachtet. Doch umgekehrt ist es nicht so, dass eine Missachtung der Autonomie von Personen auch eo ipso eine Missachtung ihrer normativen Autorität ist. Die normative Autorität wird nicht missachtet, wenn Menschen daran gehindert werden, das zu tun, was sie tun wollen. Wenn mich jemand z. B. daran hindert, eine lang geplante Reise anzutreten, missachtet er meine Autonomie. Was er tut, ist moralisch falsch, aber keine Missachtung meiner normativen Autorität. Die normative Autorität ist dem Recht, das zu tun, was man tun will, vorgelagert.

Missachtung der normativen Autorität sind Angriffe auf das Recht, über sich verfügen zu können. Sie zielen nicht auf eine Einschränkung der Autonomie von Personen, sondern darauf, ihnen klar zu machen, dass mit ihnen gemacht werden darf, was einem anderen beliebt. Die Autorität über sich wird ihnen abgesprochen. Diese Autorität geht dem Recht voraus, das zu tun, was man tun will. Der Wille von Personen ist zu achten, weil sie die normative Autorität über sich haben und d.h.: weil sie Würde haben.

\section{Sterben in Würde als selbstbestimmtes Sterben?}

Man könnte hier einwenden, dass in medizinethischen Kontexten allerdings, z. B. dort, wo von einem Sterben in Würde die Rede ist, nichts anderes gemeint ist, als ein selbstbestimmtes Sterben. Menschen können in Würde sterben, so könnte man sagen, wenn sie selber über das Ende ihres Lebens zu bestimmen in der Lage sind. Insofern könnte man in diesem Kontext auf den Begriff der Würde verzichten. Menschen ein Sterben in Würde zu ermöglichen, heißt, sie in die Lage zu versetzen, selbstbestimmt zu sterben.

\footnotetext{
${ }^{4}$ Margalit versteht Würdeverletzungen paradigmatisch als Demütigungen; vgl. [7], S. 44.
} 
Ist das richtig? Zunächst muss man feststellen, dass Unterschiedliches unter einem Sterben in Würde verstanden wird (vgl. [3]). Ich glaube aber, dass sich auch in diesem Zusammenhang ein präziser Begriff von Würde formulieren lässt, der sich von der Achtung der Autonomie unterscheidet. Sterben in Würde ist nicht mit einem selbstbestimmten Sterben gleichzusetzen.

Verschiedene Autoren meinen, mit der Würde des Menschen sei nichts anderes gemeint als die Aussage, dass menschliches Leben heilig sei. Und dies bedeute, dass menschliches Leben nicht verletzt oder zerstört, sondern immer geschützt, verteidigt und bewahrt werden soll ([5], S.203). Und weil menschliches Leben immer geschützt und nie zerstört werden dürfe, könne ein Sterben in Würde nach dieser Auffassung auch nie darin bestehen, dass man seinem Leben ein Ende setzt, oder andere darum bittet, dabei behilflich zu sein oder gar es zu beenden. Man sterbe deshalb - so z. B. Kass - nicht in Würde, wenn man sein eigenes Leben beendet oder beenden lässt, man sterbe vielmehr in Würde, wenn man ertrage, was einem das Schicksal aufträgt. ${ }^{5}$

Diese Auffassung von Sterben in Würde entspricht nicht dem, was oben als Würde des Menschen bezeichnet wurde. Wenn man Würde als normative Autorität über sich begreift, dann hat ein Sterben in Würde mit der Ausübung der normativen Autorität über sich selbst zu tun. Und das wiederum rückt die Idee würdigen Sterbens in der Tat in Richtung Autonomie. Für dieses Verständnis von Würde spricht auch der Umstand, dass das Heiligkeitsverständnis paradigmatischen Formen der Würdeverletzungen nicht Rechnung tragen kann. Es ist z. B. nicht in der Lage, die Demütigung von Menschen als Würdeverletzung zu sehen, da durch Demütigungen menschliches Leben weder zerstört noch verletzt wird. Demütigungen verletzen vielmehr Ansprüche, die Menschen haben, nicht ihr Leben.

Dennoch bedeutet dies nicht, dass ein Sterben in Würde mit einem selbstbestimmten Sterben gleichzusetzen ist. Wäre das der Fall, wäre mit einem Sterben in Würde nichts anderes als der Umstand gemeint, dass das Sterben sich nach dem freien Willen der betroffenen Person vollzieht. Das ist ein sehr weiter Begriff eines Sterbens in Würde. Es gibt gute Gründe, daran zu zweifeln, dass es dies ist, was wir mit diesem Begriff meinen. Betrachten wir dazu Folgendes: Nehmen wir an, ein junger Mann - ohne Druck und Zwang von außen - möchte, weil er in einer Liebesbeziehung tief enttäuscht wurde, seinem Leben ein Ende setzen. Wäre das ein Sterben in Würde? Und würden wir, wenn wir ihn daran hindern oder die erforderlichen Mittel nicht zur Verfügung stellen würden, ihn in seiner Würde verletzen?

Man kann natürlich auch bezweifeln, dass es sich bei dem vorliegenden Sterbewunsch um einen selbstbestimmten, autonomen Wunsch des jungen Mannes handelt. Autonom sind die Wünsche einer Person dann, wenn sie, so Holger Baumann, ,in einem emphatischen Sinne ihre eigenen sind“ ([1], S.668). Der Begriff der Autonomie ist, wie schon gesagt, umstritten. Zwar könnte der Sterbewunsch des jungen Mannes nicht selbstbestimmt sein, es kann aber auch nicht ausgeschlossen werden, dass er selbstbestimmt ist. Der junge Mann mag verzweifelt sein, aber er ist nicht deswegen schon fremdbestimmt. Auch ein Mensch, der an einer terminalen Krankheit leidet, kann verzweifelt sein und seinem Leben deswegen ein Ende setzen wollen. Man kann ihn trotzdem für autonom halten, sofern er in der Lage ist, Gründe für seine Entscheidung geltend zu machen. In gleicher Weise kann man den jungen Mann auch für autonom halten, sofern er in der Lage ist, Gründe für sein Vorhaben

\footnotetext{
5 [5], S. 218 f.: „,The deaths we most admire are those of people who, knowing that they are dying, face the fact frontally and act accordingly: [...] they continue to live and work and love as much as they can for as long as they can."
} 
vorzubringen. ${ }^{6}$ Zurück zu unserer Ausgangsfrage: Angenommen, es würde sich um einen selbstbestimmten Sterbewunsch handeln, wäre es dann auch zugleich ein Sterben in Würde?

Wenn die Würde eines Menschen in seiner normativen Autorität über sich selbst liegt, ist die Würde dann bedroht, wenn Menschen diese Autorität nicht mehr wahrzunehmen in der Lage sind. Ein Sterben in Würde hat danach damit zu tun, zu verhindern, dass man ein Leben führen muss, das keine für einen selbst akzeptablen Optionen mehr anbietet. Das hat wiederum damit zu tun, dass man über wesentliche eigene Lebensvorgänge die Kontrolle verliert oder sich aufgrund von Krankheiten ein Leben zu führen gezwungen sieht, das nicht mehr viel mit dem zu tun hat, was man zutiefst am eigenen Leben wertschätzt. Ein Sterben in Würde ist dementsprechend ein Sterben, das sich erfolgreich einer Zerstörung der normativen Autorität über das eigene Leben widersetzt.

Das jedenfalls wäre die Idee würdigen Sterbens, die sich aus dem oben explizierten Begriff der Würde ergibt. Und ich denke, dass diese Auffassung eines Sterbens in Würde auch den entsprechenden Intuitionen von vielen stärker entspricht als diejenige Auffassung, die das Sterben in Würde mit einem selbstbestimmten gleichsetzt. Die wenigsten würden es als eine Verletzung der Würde des jungen Mannes ansehen, wenn man ihm die erforderlichen Mittel zur Beendigung des eigenen Lebens nicht zur Verfügung stellen würde.

Man setzt seinem Leben dann in Würde ein Ende, wenn man sich damit einem Leben entzieht, das einem nicht mehr erlaubt, die normative Autorität über sich wahrzunehmen. Das ist der Fall, wenn man nicht mehr in der Lage ist, zwischen Optionen zu wählen, die für einen selbst akzeptabel sind. Man könnte auch sagen: Das ist der Fall, wenn man im Blick auf wesentliche Bereiche des eigenen Lebens nicht mehr das zu realisieren in der Lage ist, was einem in diesen Hinsichten wichtig ist, und man deshalb nicht mehr weiterleben möchte.

Was unterscheidet ein Sterben in Würde von einem selbstbestimmten Sterben? Ein Sterben in Würde verhindert, ein Leben führen zu müssen, in dem man die normative Autorität über sich nicht wahrzunehmen in der Lage ist. Ein selbstbestimmtes Sterben ist ein Beenden eines Lebens, das man nicht mehr leben will. Die beiden Dinge müssen nicht zusammenfallen. Es kann sein, dass ich ein Leben nicht weiterführen will, obwohl ich durchaus noch in der Lage wäre, die normative Autorität über mein Leben wahrzunehmen.

Nun könnte man argumentieren, dass ein Leben, das man nicht mehr wertschätzen kann, auch kein autonomes Leben mehr ist. Autonom lebt man bloß dann, wenn man sich für Dinge entscheiden kann, die man als akzeptabel ansieht. Man kann Autonomie ohne Zweifel so verstehen. Wenn man das tut, dann ist ein Sterben in Würde auch immer ein selbstbestimmtes Sterben. Das heißt allerdings nicht - und das ist hier wichtig - dass jedes selbstbestimmte Sterben auch ein Sterben in Würde sein muss. Man kann der Meinung sein, Gründe zu haben, sein Leben zu beenden, und dies auch wollen und gleichzeitig in der Lage sein, ein Leben zu führen, das mir Optionen bietet, die für mich akzeptabel sind. Das heißt: Man kann in der Lage sein, die eigene normative Autorität auszuüben und gleichzeitig nicht mehr weiterleben wollen. Selbstbestimmt ist ein Sterben nicht bloß dann, wenn ich nicht mehr fähig bin, das Leben zu führen, das ich führen möchte. Selbstbestimmt ist nach dem vorliegenden Vorschlag ein Sterbewunsch, für den ich Gründe geltend machen kann. Ein solcher Wunsch kann vorliegen, auch wenn ich weiterhin im subjektiven Sinn akzeptable

\footnotetext{
${ }^{6}$ Und das kann durchaus der Fall sein: Ein Leben ohne seine ehemalige Partnerin ist für ihn ohne Sinn. Er mag mit dieser Einschätzung falsch liegen. Aber das bedeutet nicht eo ipso, dass der junge Mann nicht selbstbestimmt ist, außer man hält Wünsche bloß dann für autonom, wenn sie auf keinen falschen Überzeugungen beruhen. Das wäre allerdings ein sehr anspruchsvoller Begriff von Autonomie. Die These lautet also nicht, dass solche eher unüblichen Sterbewünsche notwendigerweise selbstbestimmt sind. Sie lautet bloß, dass ein solcher Wunsch selbstbestimmt sein könnte.
} 
Optionen habe. Er mag insgesamt betrachtet unvernünftig sein, aber er ist nicht notwendigerweise fremdbestimmt. Mit Sterben in Würde ist nicht einfach ein selbstbestimmtes Sterben gemeint, sondern eines, das sich gegen ein Leben richtet, das keine akzeptablen Optionen bietet. Der Begriff des Sterbens in Würde ist enger als derjenige eines selbstbestimmten Sterbens.

Um Missverständnisse zu vermeiden, muss gesagt werden, dass Menschen ein Recht auf ein selbstbestimmtes Sterben haben könnten. Worauf Menschen ein Recht haben, ist hier nicht das Thema. Klar allerdings ist, dass eine Regelung der Sterbebeihilfe, die sich an einem Sterben in Würde orientieren würde, anders aussähe als eine, der es um den Schutz der Selbstbestimmung von Personen ginge. Und das hat seinen tieferen Grund in dem Umstand, dass eben Würde, so wie sie hier verstanden wird, einen gegenüber der Achtung vor der Autonomie eigenständigen normativen Gehalt besitzt.

\section{Ein universaler Begriff?}

Der Begriff der Würde, so die hier vertretene These, hat eine eigenständige Bedeutung, die sich nicht auf die Achtung vor der Autonomie von Personen reduzieren lässt. Aber ist dieser Begriff der Würde - so lässt sich fragen - ein universaler Begriff, der sich in allen Kulturen und Gesellschaften berechtigterweise anwenden lässt? Ist die normative Autorität über sich selbst etwas, das alle Menschen haben, unabhängig davon, in welcher Kultur sie leben und welcher Gemeinschaft sie angehören? Taugt der Begriff der Menschenwürde - anders gefragt - als Begriff einer globalen Medizinethik?

Für die Ansicht, es handle sich bei der Würde um einen universalen Begriff, spricht, dass die Zuschreibung einer normativen Autorität über sich nicht von Faktoren abhängt, die kulturabhängig sind. Wir schreiben Würde sinnvollerweise Wesen zu, die herabgesetzt und erniedrigt werden können. Wer die normative Autorität anderer angreift, tut dies, unabhängig davon, in welchem kulturellen Kontext das geschieht.

Doch nehmen wir an, es gebe Gesellschaften, die mit Herabsetzungen und Erniedrigungen nicht vertraut wären. Würden die Angehörigen dieser Gesellschaft keine normative Autorität über sich besitzen? Zunächst gilt: Menschen können erniedrigt werden, ohne die entsprechenden Handlungen als Erniedrigungen zu sehen. Generell gilt: Eine Person kann in ihrem Recht, eine normative Autorität über ihr Leben zu haben, verletzt werden, ohne dass sie das auch selber wahrnimmt. Die Verletzung der normativen Autorität einer Person ist die Verletzung eines Anspruchs, der unabhängig davon vorliegt, ob das Opfer darauf in negativer Weise reagiert. Das Opfer kann zudem darin auch kein Unrecht sehen, weil es sich selber nicht als ein Wesen, das normative Autorität über sich hat, versteht. So könnte ein Sklave sich selber alle Rechte absprechen. Das aber bedeutet nicht, dass er ein Wesen ist, das keine normative Autorität über sich besitzt. Das folgt deshalb nicht, weil die normative Autorität nichts ist, was wir uns selbst zuschreiben. Wir haben eine normative Autorität über uns, unabhängig davon, ob wir uns so sehen oder nicht.

Eine normative Autorität haben Wesen, die erniedrigt und gedemütigt werden können. Und das trifft auf alle Menschen zu. Wir sind Wesen, die erniedrigt werden können. Entsprechende Erfahrungen treffen uns in unserem Innersten. Würdeverletzungen sind ein Angriff auf das, was wir als Menschen sein wollen: Wesen, die man achtet, Wesen, über die man nicht verfügt, sondern die man als Wesen anerkennt, die ein eigenständiges Leben führen wollen. Vielleicht wollen das faktisch nicht alle Menschen. Es ist aber nicht einzusehen, dass sie keinen guten Grund haben, das zu wollen. Wer das nicht will, gibt sich als eigenständige Person auf. Niemand sollte das wollen. Wir können uns von der eigenen Würde 
nicht entbinden (vgl. [8], S. 65 ff.). So wie die Würde anderer Personen, bindet mich auch die eigene. Wir können unsere eigene Würde zwar selbst verletzen, aber nicht aufgeben. Genau deshalb kommt sie uns allen zu.

Interessenkonflikt Der Autor gibt an, dass keine Interessenkonflikte bestehen.

\section{Literatur}

1. Baumann H (2004) Autonomie und Biografie. In: Bluhm R, Nimtz C (Hrsg) Ausgewählte Beiträge zu den Sektionen der GAP.5: fünfter Internationaler Kongress der Gesellschaft für Analytische Philosophie, Bielefeld, 22.-26. September 2003. Mentis, Paderborn, S 668-678

2. Feinberg J (1986) Harm to self. Moral limits of the criminal law. Oxford University Press, Oxford

3. Gentzler J (2003) What is a death with dignity? J Med Philos 28:461-487

4. Hoerster N (2002) Ethik des Embryonenschutzes: ein rechtsphilosophischer Essay. Reclam, Stuttgart

5. Kass LN (1998) Death with dignity and the sanctity of life. In: Uhlmann M (Hrsg) Last rights?: assisted suicide and euthanasia debated. Eerdmans, Washington DC, S 199-222

6. Macklin R (2003) Dignity is a useless concept. Brit Med J 327:1419-1420

7. Margalit A (1996) The decent society. Harvard University Press, Cambridge

8. Schaber P (2010) Instrumentalisierung und Würde. Mentis, Paderborn

9. Taylor JS (Hrsg) (2005) Personal autonomy: new essays on personal autonomy and its role in contemporary moral philosophy. Cambridge University Press, Cambridge 